\title{
Effects of Online Home Nursing Care Model Application on Patients with Traumatic Spinal Cord Injury
}

\author{
Qiao-Ping $\mathrm{Li}^{1, *}$ \\ Jing $\mathrm{Li}^{\mathrm{l}}$,* \\ Hong-Ying $\mathrm{Pan}^{2}$ \\ 'Department of Spine Surgery, Lishui \\ Central Hospital, Lishui, People's \\ Republic of China; ${ }^{2}$ Department of \\ Nursing, Lishui Central Hospital, Lishui, \\ People's Republic of China \\ *These authors contributed equally to \\ this work
}

\begin{abstract}
Objective: This study aims to explore the effects of an online home nursing care model application on patients with traumatic spinal cord injury (TSCI).

Methods: Eighty patients with TSCI discharged from the hospital between January 2015 and January 2018 were included in the study. The patients were randomly divided into two groups: the control group and the observation group $(n=40$, each). The patients in the control group were given routine discharge guidance, while the patients in the observation group were given online home nursing care. The Oswestry Disability Index (ODI), Medical Outcomes Study 36-item short-form health survey (MOS SF-36), and complicationincidence rate were used to evaluate the efficiency of the online home nursing care model. Results: There were no differences in the ODI and MOS SF-36 scores between the two groups at discharge. However, the ODI and MOS SF-36 scores in the observation group showed significant improvement compared with the control group $(\mathrm{p}<0.05)$ during the most recent follow-up. The incidence of complications, such as constipation, joint stiffness, muscle atrophy, foot drop, and pressure sores, were significantly lower in the observation group than in the control group $(\mathrm{p}<0.05)$.
\end{abstract}

Conclusion: The online home nursing care model can reduce complication incidence, alleviate dysfunction, and improve the quality of life of patients with TSCI.

Keywords: online, home nursing care, spinal cord injury, Oswestry Disability Index, SF-36

\section{Introduction}

Traumatic spinal cord injury (TSCI) is one of the most common causes for disability in humans. It mainly occurs due to factors such as traffic accidents, industrial injuries, and violent incidents. ${ }^{1}$ Although progress has been made toward understanding TSCI pathogenesis and improving early recognition and treatment, TSCI still often causes serious or permanent disabilities in patients. This can seriously affect the patients' capabilities to take care of themselves and participate in social activities, making them burdens to society and their families. ${ }^{2,3}$ The incidence of TSCI-induced paraplegia in China ranges between $6.7-23.0 \%$. At present, it remains a high-risk injury. ${ }^{4}$

Patients with TSCI can master basic self-management methods after undergoing surgical rehabilitation and systematic, comprehensive training during hospitalization. However, the patients' compliance with medical treatment generally decreases after they return to their normal lives. This often results in an occurrence of complications, such as constipation, joint stiffness, muscle
Department of Nursing, Lishui Central Hospital, No. 289 of Kuocang Road, Liandu District, Lishui, People's Republic of China

Tel +8613587179166

Email panhongying_56@I63.com 
atrophy, foot drop, and pressure sores, in patients. These problems can seriously affect the patients' quality of life. ${ }^{5}$ A previous study has revealed that individualized nursing programs can lighten the load placed on the patients' caregivers and effectively improve the patients' quality of life. ${ }^{4}$ In addition, patients with TSCI suffer from long-term physical and psychological dysfunctions. Compared with other traumatic injury groups, patients with TSCI have a higher incidence of psychological problems. ${ }^{6-8}$ Various phenomena and studies have revealed that individualized out-ofhospital nursing care is essential for the rehabilitation of patients with TSCI. ${ }^{9-11}$

Online home nursing care focuses on this issue. In addition to routine in-hospital nursing care services, the internet is used to provide health education and family guidance for patients at home in order to promote their rehabilitation and improve their quality of life after discharge. ${ }^{12}$ The development of home nursing care in developed countries is advanced and includes physiological, psychological, and social nursing, medication management, etc. Subjects of home nursing care are mainly elderly patients and patients with cerebrovascular diseases, tumors, or organ transplants. Currently, home nursing care in China mainly caters to elderly patients and patients suffering from chronic diseases. ${ }^{13}$ However, a few studies on the nursing care of patients with TSCI that use the online home nursing care model have been performed. ${ }^{14}$ Considering that Lishui city is located in a mountainous area with an underdeveloped economy and limited health resources, there is no research on home nursing care application to patients with TSCI in the Lishui area. Therefore, in the present study, the effects of the online home nursing care model on the dysfunction index, quality of life, and complicationincidence in patients with TSCI are explored in order to further promote the improvement and perfection of the home nursing care model.

\section{Information and Methods}

\section{General Subject Information}

Eighty patients with TSCI discharged from our hospital from January 2015 to January 2018 were included in the present study and randomly divided into 2 groups: the control group and the observation group $(\mathrm{n}=40$, each). Patients in the control group were given routine discharge guidance, while the patients in the observation group were given online home nursing care.

Inclusion criteria: (1) patients diagnosed by imaging techniques; (2) patients with a clear history of trauma and different degrees of sensory, motor, or muscle dysfunction; (3) patients who underwent an open reduction of fracture and spinal fusion as well as an internal fixation and had good spinal stability after the operation; (4) patients without complications, such as pulmonary or urinary tract infections and pressure ulcers, at discharge; and (5) patients who provided informed consent. Exclusion criteria: (1) patients with heart, liver, or kidney dysfunctions; (2) patients with neurological diseases or brain injuries; and (3) patients with malignant tumors.

The differences in age, gender, cause and location of the injury, Frankel classification, and complication between the two groups were not statistically significant (Table 1). Furthermore, the differences in the Oswestry Disability Index (ODI) scores and the Medical Outcomes Study 36-item short-form health survey (MOS SF-36) scores between the two groups were not statistically significant.

\section{Online Home Nursing Model and Method}

Patients in both groups were given routine discharge guidance. On the basis of this guidance, the patients in the observation group adopted the online home nursing care model. Specific measures were as follows: (1) the establishment of a database of TSCI patients containing diagnoses, admission numbers, Frankel spinal cord injury classifications, contact modes, caregiver statuses, family economic conditions, community support, complications, changes in the dysfunction index, and quality-of-life improvements during the follow-up period. (2) The establishment of a systematic health education process: (A) the patients were educated about the entire process of the disease immediately after admission; (B) the patients were provided with strengthening guidance before discharge. Three days before discharge, the doctor in charge provided ideological education to the main caregivers to increase their nursing awareness and improve their compliance behavior. The responsible nurses trained the patients in relevant skills and provided video education for both the patients and their families, a hands-on demonstration of various factors that can lead to urethral obstruction, muscle atrophy, joint stiffness, foot drop, or pressure sores, intervention 
Table I Clinical Data of 80 Patients Enrolled in the Study

\begin{tabular}{|c|c|c|c|c|}
\hline Index & Control Group (40 Cases) & Observation Group (40 Cases) & $t / X^{2}$ & $\boldsymbol{P}$ \\
\hline Age & $17 \sim 69(59.1)$ & $23 \sim 72(57.8)$ & 0.61 & 0.543 \\
\hline Cause of the injury & - & - & 0.00 & 1.000 \\
\hline Traffic accident injury & 19 & 22 & - & - \\
\hline Falling injury & 15 & 13 & - & - \\
\hline Heavy object injury & 6 & 5 & - & - \\
\hline Gender & - & - & 0.00 & 1.000 \\
\hline Male & 26 & 28 & - & - \\
\hline Female & 14 & 12 & - & - \\
\hline Location of the injury & - & - & 0.00 & 1.000 \\
\hline Cervical segment & 15 & 13 & - & - \\
\hline Thoracic segment & 19 & 18 & - & - \\
\hline Lumbar segment & 6 & 9 & - & - \\
\hline Frankel classification & - & - & 0.00 & 1.000 \\
\hline A & 6 & 5 & - & - \\
\hline B & 12 & 10 & - & - \\
\hline C & 11 & 13 & - & - \\
\hline $\mathrm{D}$ & 7 & 7 & - & - \\
\hline E & 4 & 5 & - & - \\
\hline Complications & - & - & 0.894 & 0.465 \\
\hline Primary hypertension & 9 & 6 & - & - \\
\hline Diabetes & 5 & 4 & - & - \\
\hline
\end{tabular}

Notes: Data were analyzed using statistical software SPSSI7.0. Measurement data were expressed as mean \pm standard deviation ( $x \pm$ SD), and compared between the two groups using the $t$-test. Count data were expressed as a (\%) rate and compared between groups using the $X^{2}$ test. $P<0.05$ was considered statistically significant.

measures, and functional exercise methods, until the main caregivers could master and operate the knowledge and skills; and (C) the patients attended a follow-up after discharge. A hope house and home nursing care team consisting of physicians, nurses, rehabilitation therapists, psychological consultants, and nutritionists were established. One week after discharge, the home nursing care team used weekend time to conduct on-site rehabilitation nursing guidance at the patients' homes. The outpatient reviews were conducted three months later, and each patient underwent a follow-up conducted by the community four months later. (3) The establishment of an internet platform utilizing WeChat, a mobile client, telephone, Micro-blog, and QQ group. Advisory services, such as nursing, medical treatment, prevention, and health care, were supplied through multiple channels in order to provide home medical and nursing services and meet the difficult medical treatment needs in mountainous areas. The online nursing model comprises a WeChat group, public account, microblog, QQ, etc., where nurses and doctors regularly push health knowledge and answer patients' questions at any time. The WeChat group, QQ, and health knowledge database are regularly updated on the public account and microblog. The nurses who collected patient data received homogenization training before doing so. ODI and MOS SF-36 questionnaires were sent to the present study's patients to fill in, after which they were reviewed by the nurses. The patients who were not able to fill in the questionnaires were asked by the nurses to fill in item by item.

\section{Effectiveness Evaluation Method}

The follow-up data were analyzed retrospectively. The differences in the patient complication incidence, dysfunction index, and quality of life were compared between the two groups at 12 months after discharge. The specific data were as follows: (1) complications constipation, joint stiffness, muscle atrophy, foot drop, and pressure sores - were assessed following six months of nursing after discharge; (2) dysfunction: the patients were assessed using the ODI questionnaire 
at admission and 12 months after discharge. The contents included nine aspects (pain intensity, self-care, objects lifting, walking, sitting, standing, sleep disturbance, social life, and tourism). The item "sexual life" was not included. Six options were set for each question. The first option scored zero points, and the last option scored five points. The higher the score, the more serious the dysfunction; (3) quality of life after 12 months of nursing following discharge, the patients were assessed via face-to-face consultation. The quality of life was assessed using the MOS SF36 questionnaire, which worked with 8 aspects (physical function, physical role, body pain, general health status, vitality, social function, emotional role, and mental health). The higher the score, the higher the quality of life.

\section{Statistical Analysis}

Data were analyzed using the Statistical Package for Social Sciences 17.0. Measurement data were expressed as the mean \pm standard deviation and compared between the two groups using the $t$-test. Count data were expressed as a percentage rate and compared between the groups using the $\mathrm{X}^{2}$ test. A $\mathrm{p}$ value of $<0.05$ was considered statistically significant.

\section{Results}

\section{Incidence of Complications in Two Groups of Patients}

The number of cases of pressure sores, constipation, joint stiffness, muscle contracture and foot drop in the control group were $11(27.5 \%), 6$ (15\%), 7 (17.5\%), 4 $(10 \%)$ and $4(10 \%)$, respectively, and the number of cases of pressure sores, constipation, joint stiffness, muscle contracture and foot drop in the observation group were $2(5 \%), 1(2.5 \%), 1(2.5 \%), 0(0 \%)$ and 0 $(0 \%)$, respectively. All complications were fewer in the observation group than in the control group. The differences between the two groups were statistically significant (Table 2).

\section{Comparison of ODI Between the Two Groups}

There was no difference in ODI between the two groups at discharge $(\mathrm{P}>0.05)$. At the latest follow-up, the ODI was significantly lower in the observation group than in the control group $(22.9 \pm 5.0$ vs $30.1 \pm 4.44)$. The difference between the two groups was statistically significant (Table 3).

\section{Comparison of the Quality of Life Between the Two Groups}

There was no difference in SF-36 between the two groups at discharge $(\mathrm{P}>0.05)$. At the latest follow-up, the SF-36 was significantly higher in the observation group than in the control group $(75.8 \pm 6.0$ vs $63.2 \pm 7.8)$. The difference between the two groups was statistically significant (Table 4).

Table 3 Comparison of ODI Between the Two Groups

\begin{tabular}{|l|c|c|}
\hline Group & Number of Cases & ODI \\
\hline Control group & 40 & $30.1 \pm 4.44$ \\
\hline Observation group & 40 & $22.9 \pm 5.0$ \\
\hline$t$ & - & -6.781 \\
\hline$P$ & - & 0.000 \\
\hline
\end{tabular}

Notes: Data were analyzed using statistical software SPSS 17.0. Measurement data were expressed as mean \pm standard deviation $(x \pm S D)$, and compared between the two groups using the $t$-test. Count data were expressed as a (\%) rate and compared between groups using the $X^{2}$ test. $P<0.05$ was considered statistically significant.

Table 2 Incidence of Complications in Two Groups of Patients

\begin{tabular}{|l|c|c|c|c|c|c|}
\hline Group & Number of Cases & Pressure Sores & Constipation & Joint Stiffness & Muscle Contracture & Foot Drop \\
\hline Control group & 40 & II & 6 & 7 & 4 & 4 \\
\hline Observation group & 40 & 2 & I & I & 0 & 0 \\
\hline$\chi^{2}$ & - & 7.44 & 3.914 & 5.000 & 4.211 & 4.211 \\
\hline$P$ & - & 0.006 & 0.048 & 0.025 & 0.04 & 0.04 \\
\hline
\end{tabular}

Notes: Data were analyzed using statistical software SPSSI7.0. Measurement data were expressed as mean \pm standard deviation $(x \pm$ SD), and compared between the two groups using the $t$-test. Count data were expressed as a (\%) rate and compared between groups using the $X^{2}$ test. $P<0.05$ was considered statistically significant. 
Table 4 Comparison of the Quality of Life Between the Two Groups

\begin{tabular}{|l|c|c|}
\hline Group & Number of Cases & SF-36 \\
\hline Control group & 40 & $63.2 \pm 7.8$ \\
\hline Observation group & 40 & $75.8 \pm 6.0$ \\
\hline$t$ & - & 8.112 \\
\hline$P$ & - & 0.000 \\
\hline
\end{tabular}

Notes: Data were analyzed using statistical software SPSSI7.0. Measurement data were expressed as mean \pm standard deviation $(x \pm S D)$, and compared between the two groups using the $t$-test. Count data were expressed as a (\%) rate and compared between groups using the $X^{2}$ test. $P<0.05$ was considered statistically significant.

\section{Discussion}

\section{The Online Home Nursing Care Model Can Reduce Complication-Incidence in Patients with TSCl}

Table 2 reveals that the complication-incidence was significantly lower in the observation group than in the control group. It was revealed in interviews with the family members that after discharge, the family members of the patients in the control group did not have sufficient knowledge of the complications and were not aware of possible dangers. The assistance of the caregiver was limited to general, daily care, and the patients visited a doctor only in the case of not having an alternative. In the observation group, the rehabilitation nursing skills continued to be carried out after discharge and good compliance behavior was formed.

Patients with early symptoms of discomfort were able to get help from medical staff through the abovementioned internet platforms as well as receive encouragement and mutual assistance from friends at the hope house, helping them avoid aggravation caused by their conditions. In the current study, several patients with TSCI were found to have early-stage reddened, sacrococcygeal skin. They took photos of the reddened skin, uploaded them via WeChat for consultation, and effectively underwent decompression, thus, avoiding the occurrence of pressure sores. During a home nursing team visit, it was discovered that one patient had a stage II pressure sore on the hip and another patient had a stage III pressure sore on the heel. After decompression guidance and a targeted, on-site dressing change, both patients were successfully healed.

\section{The Online Home Nursing Care Model Can Alleviate Dysfunction in Patients with TSCI}

Table 3 reveals that the ODI and MOS SF-36 scores were significantly lower in the observation group than in the control group. The ODI assesses the degree of dysfunction in patients with spinal injury based on the patients' pain and self-care, walking, and sitting abilities scores. ${ }^{15}$ MOS SF-36 assesses the patients' quality of life based on social interaction, daily activities, and emotions. ${ }^{16}$ A previous study reveals that disuse syndrome might occur when joint dysfunction lasts only 1-2 months. The study shows that when it lasted over 6 months, functional exercise had little effect. An occurrence of complications, such as joint stiffness, muscle atrophy, and foot drop, within 3 months caused obstacles in the next steps of the rehabilitation process. ${ }^{17}$ In the control group, nursing was basically reliant on the caregivers. Lacking effective guidance and communication, patients became lazy in terms of recovery over time. The internet is an open platform that is well suited for the purpose of gaining information. In the observation group, the patients could learn rehabilitation skills in the group space and from group announcements, sharing, videos, pictures, and words, in addition to getting answers to their questions. An early intervention of the home nursing team, enabled by friends or patient circles sharing their experiences and communicating their insights, can help gain a better understanding of the needs of patients with TSCI and their families and aid the barrier-free transformation of patients' living environments by creating convenient living facilities, providing continuous interventions, and strengthening the patients' self-care abilities in daily life, thus alleviating their dysfunctions.

A previous study also reveals that online home nursing care for patients with diabetes could significantly improve patients self-management, body mass index, blood glucose, and daily mean blood glucose fluctuation. This method has significant advantages compared with conventional diabetes management methods. It is also conducive to diabetes control. These results suggest that online home nursing care will also have advantages compared with the traditional care model. ${ }^{18}$ The results of this study are similar to the results of previous studies. 


\section{The Online Home Nursing Care Model Can Improve the Quality of Life of Patients with TSCl}

With the passage of time, the adaptability of patients with TSCI to life decreases in the face of physiological changes. In addition, they are confronted with family dependence, physical pain, and economic burdens, all of which may result in psychological problems, such as depression. The network used in the online home nursing care model is an open communication platform that can effectively break through the restrictions of the mountainous region. People's way of life is greatly affected by time and economy and everyone can get a certain amount of out-of-hospital knowledge through chatting and consulting. For example, the utility characteristics of several auxiliary devices can be shared, providing a fast rehabilitation nursing service in which continuous rehabilitation guidance can be accessed from home. Some social activities of the hope house have given the patients positive energy and significantly improved their quality of life. This is confirmed by the results of the present study.

The significant effect that the online home nursing care model has on patients has also been observed in studies on other diseases. For example, the nursing satisfaction score after discharge was significantly higher in patients who had experienced a stroke in the observation group than in the control group. Furthermore, anxiety, depression, and quality of life scores were significantly better in the observation group than in the control group. Therefore, we can conclude that the online home nursing care model has the ability to effectively improve the psychological and physiological status of patients who have experienced a stroke, which has a high clinical-popularization value. ${ }^{19}$

\section{Promotion in the Medical Service Field}

Today's society is situated in the information age. Mobile internet encompasses the largest user group of mobile instant messaging software in Asia and offers strong interaction, low cost, and fast speed. The online home nursing care model avoids unnecessary back-and-forth running between the hospital and the homes of patients with TSCI. It is a part of the "one run at most" reform in the medical service field. It not only saves manpower, materials, and financial resources, but also reduces the long-term occupancy of hospital beds caused by re-admission, alleviates the burdens placed upon society and the patients' families, and meets follow-up rehabilitation needs outside the hospital. It also has great social and economic benefits.
Yang et $\mathrm{al}^{20}$ uses the "Internet + " management service model for the treatment of patients with hypertension. The service model significantly reduces the systolic blood pressure levels and improves the blood pressure control rates. Studies have shown that ${ }^{21}$ health education based on the internet + community model has a significant effect on the prevention of osteoporosis. Furthermore, ${ }^{22}$ based on internet + , the health management model's three-way linkage of the hospital, community, and family can increase the patients' self-energy management, reduce disease symptoms, and improve patient satisfaction. The region included in the present study is located in a mountainous area, meaning that when complications occur, patients with limited limb function cannot seek medical treatment in time. After the application of online nursing, patients can consult their issues via the internet at any time, reducing complication occurrence.

\section{Thinking and Limitations}

The city included in the present study is located in a mountainous area with an underdeveloped economy, causing a long course of affliction for patients with TSCI. Different environments and the level of the support from the patients' families will inevitably affect rehabilitation effectiveness. At present, the technical strength of community health service centers is low, and tertiary and community hospitals do not achieve seamless docking. Thus, they cannot provide highquality specialized nursing. Third-level hospitals have insufficient medical and nursing manpower to arrange full-time online questions and answers. Furthermore, they lack longterm service. The psychological aspects of the patients were not assessed and intervened in this study. However, it is a possible direction for further research. No psychological evaluation was conducted in this study.

\section{Ethics Approval and Consent to Participate}

All the authors confirm this study was conducted with approval from the Ethics Committee of Lishui Central Hospital. A file copy can be provided for review if required. Written informed consent was obtained from all participants.

This study was conducted in accordance with the declaration of Helsinki.

\section{Funding}

Lishui Science and Technology Project, Effect of Continuous Nursing on Family Function and Health 
Behavior of Patients with Thoracolumbar Fracture Complicated with Spinal Cord Injury, Item Edition: 2017GYX06, Construction and reliability test of selfmanagement scale for patients with spinal cord injury (2021RC037).

\section{Disclosure}

The authors report no conflicts of interest in this work.

\section{References}

1. Sarwahi V, Galina J, Hasan S, et al. A study of critical events that lead to spinal cord injury and the importance of rapid reversal of surgical steps in improving neurological outcomes: a porcine model. Spine (Phila Pa 1976). 2019. doi:10.1097/BRS.0000000000003229

2. Kashif M, Jones S, Darain H, Iram H, Raqib A, Butt AA. Factors influencing the community integration of patients following traumatic spinal cord injury: a systematic review. J Pak Med Assoc. 2019;69(9):1337-1343.

3. Naduvanahalli Vivekanandaswamy A, Kannan M, Sharma V, et al. Prognostic utility of magnetic resonance imaging (MRI) in predicting neurological outcomes in patients with acute thoracolumbar spinal cord injury. Eur Spine J. 2019;29(6):1227-1235. doi:10.1007/ s00586-019-06135-6

4. Blanco Belver A, Aach M, Schmiegel W, Schildhauer TA, Meindl R, Brechmann T. Similar adenoma detection rates in colonoscopic procedures of patients with spinal cord injury compared to controls. Dig Dis Sci. 2019;65(4):1197-1205. doi:10.1007/s10620-019-05814-0

5. Wang S, Smith GM, Selzer ME, Li S. Emerging molecular therapeutic targets for spinal cord injury. Expert Opin Ther Targets. 2019;23 (9):787-803. doi:10.1080/14728222.2019.1661381

6. Rezaei M, Sharifi A, Vaccaro AR, Rahimi-Movaghar V. Home-based rehabilitation programs: promising field to maximize function of patients with traumatic spinal cord injury. Asian $J$ Neurosurg. 2019;14(3):634-640. doi:10.4103/ajns.AJNS_86_17

7. Savikj M, Kostovski E, Lundell LS, Iversen PO, Massart J, Widegren U. Altered oxidative stress and antioxidant defence in skeletal muscle during the first year following spinal cord injury. Physiol Rep. 2019;7(16):e14218. doi:10.14814/phy2.14218

8. Hayashi T, Fujiwara Y, Sakai H, et al. The time course of dysphagia following traumatic cervical spinal cord injury: a prospective cohort study. Spinal Cord. 2019. doi:10.1038/s41393-019-0347-4

9. Shabany M, NikbakhtNasrabadi A, Mohammadi N, Pruitt SD. Familycentered empowerment process in individuals with spinal cord injury living in Iran: a grounded theory study. Spinal Cord. 2019;58 (2):174-184. doi:10.1038/s41393-019-0348-3
10. Richard-Denis A, Chatta R, Thompson C, Mac-Thiong JM. Patterns and predictors of functional recovery from the subacute to the chronic phase following a traumatic spinal cord injury: a prospective study. Spinal Cord. 2019. doi:10.1038/s41393-019-0341-x

11. Malik RN, Eginyan G, Lynn AK, Lam T. Improvements in skilled walking associated with kinematic adaptations in people with spinal cord injury. J Neuroeng Rehabil. 2019;16(1):107. doi:10.1186/ s12984-019-0575-z

12. Mcleod JC, Diana H, Hicks AL. Sprint interval training versus moderate-intensity continuous training during inpatient rehabilitation after spinal cord injury: a randomized trial. Spinal Cord. 2019;58 (1):106-115. doi:10.1038/s41393-019-0345-6

13. Mneimneh F, Moussalem C, Ghaddar N, Aboughali K, Omeis I. Influence of cervical spinal cord injury on thermoregulatory and cardiovascular responses in the human body: literature review. J Clin Neurosci.2019;69:7-14. doi:10.1016/j.jocn.2019.08.022

14. Kryger MA, Crytzer TM, Fairman A, et al. The effect of the interactive mobile health and rehabilitation system on health and psychosocial outcomes in spinal cord injury: Randomized Controlled Trial. $J$ Med Internet Res. 2019;21(8):e14305. doi:10.2196/14305

15. Pennings JS, Devin CJ, Khan I, Bydon M, Asher AL, Archer KR. Prediction of Oswestry Disability Index (ODI) using PROMIS-29 in a national sample of lumbar spine surgery patients. Qual Life Res. 2019;28(10):2839-2850. doi:10.1007/s11136-019-02223-8

16. Karmisholt J, Andersen S. Detecting true change in the hospital anxiety and depression scale, SF-36, and hypothyroid score when monitoring patients with subclinical hypothyroidism. Eur Thyroid J. 2019;8(3):144-151. doi:10.1159/000496827

17. Kunz S, Fellinghauer C, Peter C. Measuring posttraumatic growth and depreciation after spinal cord injury: a Rasch analysis. Rehabil Psychol. 2019;64(4):407-424. doi:10.1037/rep0000288

18. Chamberlain JD, Brinkhof MWG. Using strong inference to answer causal questions in spinal cord injury research. Spinal Cord. 2019;57 (11):907-908. doi:10.1038/s41393-019-0344-7

19. Gong Z, Xia K, Xu A, et al. Stem cell transplantation: a promising therapy for spinal cord injury. Curr Stem Cell Res Ther. 2020;15 (4):321-331. doi:10.2174/1574888X14666190823144424

20. Yang YJ, Pan D, Chang J, et al. The effect of "Internet +" on blood pressure management in patients with hypertension. Chin J Cardiovasc Res. 2019;18(5):430-434.

21. Chen J, Wang L, Wang SX, et al. Effect of "Internet + community" health education mode on prevention of osteoporosis in 78 cases. Hunan JTradit Chin Med. 2020;36(9):130-132.

22. Wang CY, Yin M, Zhang J, et al. The application of hospital, community and family combined health management mode based on "Internet +" in patients with peptic ulcer. J Nurs. 2020;35(15):96-98.
Risk Management and Healthcare Policy

\section{Publish your work in this journal}

Risk Management and Healthcare Policy is an international, peerreviewed, open access journal focusing on all aspects of public health, policy, and preventative measures to promote good health and improve morbidity and mortality in the population. The journal welcomes submitted papers covering original research, basic science, clinical \& epidemiological studies, reviews and evaluations, guidelines, expert opinion and commentary, case reports and extended reports. The manuscript management system is completely online and includes a very quick and fair peer-review system, which is all easy to use. Visit http://www.dovepress.com/testimonials.php to read real quotes from published authors. 